\title{
A Survey-based Approach for Selecting the Stations and Links for a Rapid Transit Network
}

\author{
L.F. Escudero ${ }^{1}$, S. Muñoz ${ }^{2}$ * \\ ${ }^{1}$ Departamento de Estadística e Investigación Operativa, Universidad Rey Juan Carlos, \\ 28933 Móstoles (Madrid), Spain \\ E-mail: laureano.escudero@urjc.es \\ ${ }^{2}$ Instituto de Matemática Interdisciplinar (IMI), \\ Departamento de Estadística e Investigación Operativa, Universidad Complutense de Madrid, \\ Facultad de Ciencias Matemáticas, \\ 28040 Madrid, Spain \\ E-mail:smunoz@estad.ucm.es
}

Received 19 December 2012

Accepted 3 September 2013

\begin{abstract}
In this work we present the first stage of a two-stage approach for designing rapid transit networks, where the stations and links to be constructed are selected by solving an integer linear optimization model that maximizes an estimation of the number of trips through the rapid transit network. It is based on the first stage of another approach that we described elsewhere. Instead of considering a static origin-destination matrix, we propose a novel way of computing the expected number of trips by making use of the results from a survey amongst the potential users of the rapid transit network. We also report some computational experiments on several randomly generated instances. In the second stage of the approach, a set of lines is generated for the rapid transit network.
\end{abstract}

Keywords: Station and Link Location, Transportation Systems, Survey, Degree of a Node, Optimization

\section{Introduction}

As the population concentration increases in urban areas, it gets necessary to either develop new transportation systems or to improve and/or expand the existing ones. There are so many factors to be taken into consideration to tackle these problems, that the resulting mathematical optimization models would be too complex to be solved in an exact way. Consequently, it is required to resort to simplifications and heuristic procedures.
Several authors have dealt with these problems, focussing them mainly on two opposite aims: to achieve a high service quality with affordable operating costs, or to reduce as much as possible the operating costs while maintaining a certain service quality level. Herein we focus on the first aim; some works focussing on the second aim are Claessens et al. ${ }^{1}$, Bussieck et al. ${ }^{2}$ and Goossens et al. ${ }^{3}$.

In order to illustrate the great diversity of the approaches that can be found in the literature, some works are outlined below. A point in common for

${ }^{*}$ Corresponding author 
all of them is the consideration of a static origindestination (O-D) matrix which contains the demand for each O-D pair of station locations.

Mandl ${ }^{4}$ proposes a heuristic algorithm for improving an existing public transportation network of streets or rails in an urban area, in case of absence of capacity constraints for utilizing the network. The algorithm starts with a feasible set of lines (i.e., a set of lines such that all station locations belong to at least one line and all pairs of station locations are mutually reachable), and iteratively searches for new feasible sets of lines which lead to a reduction of the total average transportation cost of the passengers. The transportation cost is defined as a weighted sum of the waiting, travel and transfer costs, which can be interpreted as time, and it is assumed that each passenger utilizes a path that minimizes his or her average transportation cost. The total average transportation cost is estimated from a given O-D matrix. The vehicle assignment problem is also briefly discussed.

Given a set of potential bus station locations and a set of potential links between them, and given a symmetric O-D matrix, Baaj and Mahmassani ${ }^{5}$ describes an Artificial Intelligence-based approach for determining a set of lines and their associated frequencies attempting to reduce the number of passengers that require any transfer to arrive at their destinations, so that both the percentages of passengers that require no transfer and of those that require a maximum of two transfers, are greater or equal to certain prespecified values. The approach consists of three major components: a line generation design algorithm, an analysis procedure, and a line improvement algorithm. It is assumed that each passenger utilizes a path that involves the fewest possible number of transfers.

Given a set of potential lines for a railway system, Bussieck et al. ${ }^{6}$ provides an integer linear optimization model for selecting a subset of these lines and determining their frequencies, with the goal of maximizing the number of passengers that require no transfer to arrive at their destinations. It assumes the symmetry of the O-D matrix as well as that each passenger utilizes a shortest path (with respect to some measure) between his or her origin and des- tination.

Guan et al. ${ }^{7}$ proposes a 0-1 linear optimization model for simultaneous optimization of transit line configuration and passenger line assignment. Starting from a given set of station locations and links between them, its aim is to select the lines to be constructed and to assign a path in the resulting network to the passengers of each O-D pair of station locations, in such a way that the union of the selected lines contains all the given links, and that a weighted sum of the total length of the lines, the total number of lines used by the passengers and the total distance covered by the passengers is minimized. For this purpose, a pool of potential lines to be selected and a pool of potential paths to be assigned to the passengers of each O-D pair are considered, and each one of these potential lines is assigned a prefixed frequency. Moreover, it is assumed that the cost of operating any line is linearly proportional to the length of that line, that each passenger utilizes a path that minimizes his or her expected travel time, and that finding the smallest number of transfers for the path assigned to the passengers of each O-D pair is equivalent to finding the smallest number of lines that those passengers should use. Neither the waiting time for the passengers nor the effect of passenger crowding are taken into consideration.

Marín ${ }^{8}$ states the extended rapid transit network design problem and provides a 0-1 linear optimization model for solving it. Given a set of potential station locations and a set of potential links between them, this problem basically consists in selecting which stations and links to construct without exceeding the available budget, and determining an upper bounded number of noncircular lines from them, to maximize the expected total number of trips through the rapid transit network, which is computed from a given O-D matrix and a given private transportation cost for each O-D pair of station locations. It is assumed that each user will utilize the rapid transit network if and only if there is any path in this network between his or her origin and destination such that its length is less or equal to the corresponding transportation cost in the private transit network. Similar models are considered in Laporte et al. ${ }^{9,10}$. 
Marín and García-Ródenas ${ }^{11}$ presents a nonlinear optimization model for locating the infrastructure of a rapid transit network without exceeding the available budget. Two alternative objective functions are proposed, namely, the expected total number of trips through the rapid transit network (to be maximized), and the expected total transportation cost through an existing private transit network (to be minimized). Both of them are defined from a given O-D matrix, a given private transportation cost for each O-D pair of station locations, and the Logit function, which is approximated by a piecewise linear function. As a consequence of this approximation, the initial nonlinear model results in an integer linear optimization model. Among the considered assumptions to simplify the model are that there is no waiting time for the users and there are no capacity constraints for utilizing the network. The model also includes some constraints that avoid the definition of circular lines, and others that attempt to minimize the number of lines to be constructed. The potential users' behavior is modeled by means of the Logit function, instead of considering the all-ornothing model from Marín ${ }^{8}$.

Escudero and Muñoz ${ }^{12}$ provides a two-stage approach for solving a modification of the extended rapid transit network design problem to allow the definition of circular lines, and shows that it outperforms the solving of a modification of the model given in Marín ${ }^{8}$ to adapt it to this new problem. In the first stage of the proposed approach, an integer linear optimization model is solved for selecting the stations and links to be constructed without exceeding the available budget, so that the expected total number of trips through the rapid transit network is maximized (without loss of generality, it is assumed that whichever two station locations are linked by one line at most). In the second stage the line design problem is solved by assigning each selected link to exactly one line, in such a way that the number of lines that go to each selected station location is as small as possible (no upper bound for the number of lines is required).

Escudero and Muñoz ${ }^{13}$ proposes some improvements on the approach stated in Escudero and Muñoz ${ }^{12}$, which, as well as the approach given in
Marín ${ }^{8}$, neither guarantees a connected rapid transit network nor takes transfers into account. On one side, it introduces several modifications in the model considered in the first stage to obtain a connected rapid transit network and to deal with potential locations for the stations, resulting in a nonlinear integer optimization model that can be linearized by introducing some additional $0-1$ variables and replacing some of the initial constraints with new ones. On other side, it presents a greedy heuristic procedure which is a modification of the algorithm proposed for solving the line design problem of the second stage. This new procedure attempts to minimize an estimation of the total number of transfers that should be made by the users to arrive at their destinations, without increasing the number of lines going to each selected station location.

Another point in common for the above works is that all of them make the explicit or implicit assumption that the users obey the well-known Wardrop's first principle (see Wardrop ${ }^{14}$ ), and, hence, they seek to minimize their expected travel costs (the above works interpret the travel cost in different ways).

In this work we tackle the problem of determining the stations and links to be constructed for a rapid transit network. The procedure that we present is based on the first stage of the approach given in Escudero and Muñoz ${ }^{13}$. Escudero and Muñoz ${ }^{15}$ contains a preliminary version of this work.

As in the above works, we also assume that the users obey Wardrop's first principle of route choice, where the travel cost is interpreted as the travel time. When dealing with road networks, instead of making this assumption it is usual to consider a bilevel optimization model to account for the route choice behaviors of the users (see e.g. Gao et al. ${ }^{16}$ and Zhang and $\mathrm{Gao}^{17}$ ). However, we are already dealing with a very complex problem, which would result in a much more complex problem if we considered a bilevel optimization model.

In this paper we present the first stage of an approach for designing rapid transit networks, where an integer linear optimization problem is solved for selecting the stations to be constructed and the links between them, considering a budget for the total 
construction cost. All of the station locations are assumed to be known, but we distinguish between key and non-key stations: the key stations have to be mandatorily constructed and they may belong to more than one line, whereas the non-key stations are always located on some link joining two key stations, and they are constructed if and only if that link is constructed.

Obviously, the solution obtained will strongly depend on the objective function considered; thus, an appropriate choice of the objective function is crucial for getting a successful rapid transit network. Among the different objective functions considered in the works outlined above, the one more directly related to the service quality is the expected total number of trips through the rapid transit network, since the higher the service quality, the greater the total number of trips. Therefore, we are considering it as the objective function (to be maximized).

This type of objective function has already been considered in Marín ${ }^{8}$, Marín and GarcíaRódenas ${ }^{11}$, Escudero and Muñoz ${ }^{12,13}$ and Laporte et al. ${ }^{9,10}$, and its value has been computed by considering a unique transportation cost for each O-D pair of station locations in an alternative transit network. This way of computing the objective function value is not very accurate, since the users of each O-D pair can actually utilize distinct means of transportation and distinct routes to arrive at their destinations, hence it does not seem adequate to consider the same alternative transportation cost for all of them. Instead, we propose to perform a survey in order to collect certain data which will make it possible to consider each potential user's behavior individually and, as a consequence, to compute the objective function value more accurately.

In the second stage of the approach, the line design problem is solved.

It is worth noting that, even though both stages of this approach make use of certain information from the survey, the two stages are independent one from the other, since, given the stations and links to be constructed that are obtained in the first stage, the line design does not have to be necessarily determined by applying the procedure considered in the second stage, and, reciprocally, in order to apply this line design procedure, the stations and links to be constructed are not required to be obtained by the method considered in the first stage.

The remainder of the paper is organized as follows: Section 2 states the basic notation and assumptions that we consider. Section 3 proposes an integer linear optimization model for selecting the stations and links to be constructed. Section 4 describes the procedure we have followed for randomly generating the tested example cases and reports some computational experience on these cases. Finally, Section 5 draws some conclusions and future research from this work.

\section{Basic Notation and Assumptions}

Let us consider two types of stations: key stations and non-key stations. The key stations will be located on the busiest zones of the area covered by the rapid transit network, which are assumed to be known (see in Laporte et al. ${ }^{18}$ a procedure for selecting such key station locations based on partitioning into zones the area under consideration and quantifying the number of trips that each zone will produce or attract). We also assume that the potential links between the key station locations are known, and that some other stations, called non-key stations, can be located on those links, in such a way that each non-key station will be constructed if and only if the link on which it lies is constructed.

The key station locations will be represented as the nodes of a graph, and the potential links between them as the edges of that graph (it is not necessary to represent the non-key station locations). Thus, we are implicitly assuming that, for each pair of distinct key station locations that can be linked, the route followed by the users for going from one of the locations to the other one will be the same as for going from the second location to the first one, but in the opposite direction. Although this assumption is usually satisfied for rail networks, it can be violated for street networks containing one-way streets, but the presented approach will remain valid for this case by representing those streets by arcs, and by modifying it accordingly. 
The notation below can be found listed and summarized in Appendix A.

Let $V=\{1, \ldots, n\}$ be the set of key station locations, let $E$ be the set of (nonordered) pairs of key station locations that can potentially be linked, i.e., $E=\{\{i, j\} \in V \times V \mid i \neq$ $j$ and it is possible to link $i$ and $j\}$, and let $m=|E|$.

Let us consider the simple graph $G=(V, E)$. Without loss of generality, whenever we refer to an edge $\{i, j\} \in E$ it will be assumed that $i<j$. We also assume that $G$ is connected.

For each $i \in V$, let $a_{i}$ be the cost of constructing a key station at $i$, and let $\Gamma(i)$ be the set of key station locations that can be linked to $i$ (notice that $\Gamma(i)$ is the set of nodes adjacent to $i$ in $G$ and $|\Gamma(i)|$ is the degree of $i$ in $G$ ).

For each $\{i, j\} \in E$, let $d_{i j}$ be the length of link $\{i, j\}$ (expressed in kilometers), let $s_{i j}$ be the number of non-key station locations on link $\{i, j\}$, and let $c_{i j}$ be the cost of linking $i$ and $j$ (including the cost of constructing the corresponding non-key stations).

If there were $\lambda$ lines going to a key station location $i$ or linking two key station locations $i$ and $j$, then the associated construction costs would be $\lambda a_{i}$ and $\lambda c_{i j}$, respectively, since it is assumed that we construct as many stations at $i$ and as many links between $i$ and $j$ as the number of lines involved. Although in the first stage of the proposed approach we implicitly consider that whichever two station locations are linked by one line at most (see Section 3), in the second stage we shall allow pairs of station locations linked by more than one line (see Sections 6 , 7 and 8 of Escudero and Muñoz ${ }^{15}$ ).

Let $b$ be the available budget for constructing the rapid transit network, and let $\bar{v}$ be the average velocity of the network's vehicles (expressed in kilometers per hour).

For each $i \in V$, let $\bar{t}(i)$ be the average time required for going between the entrance of the key station located at $i$ and its boarding and alighting platform (expressed in minutes).

Let $\bar{t}_{a}$ be the average interarrival time (i.e., the time difference between two consecutive arrivals) of the vehicles at each station (expressed in minutes), let $\bar{t}_{s}$ be the average dwell time (i.e., the time spent for boarding and alighting of passengers) of the vehicles at each station (expressed in minutes), and let $\bar{t}_{r}$ be the average time for making a transfer (expressed in minutes). Since in real-life rapid transit networks most of the time there are no vehicles at the stations, it will be assumed that $\bar{t}_{s}<\frac{\bar{t}_{a}}{2}$. If we had appropriate a priori information, instead of considering a unique value for $\bar{t}_{r}$, we could make it depend on the key station locations, i.e., we could consider the average time for making a transfer at each $i \in V$.

Let $W=\{(i, j) \in V \times V \mid i<j\}$, and let us denote $w=\left(e_{w}, e_{w}^{\prime}\right) \quad \forall w \in W$ (W is understood as the set of all distinct pairs of key station locations).

In order to assess the behavior pattern of the potential users, we propose to survey a sample of people who, a priori, are willing to utilize the rapid transit network. Let $\Theta$ be the set of surveyed people.

For each $\theta \in \Theta$ and for each $w \in W$, let $\alpha_{w}(\theta)$ be the number of trips in a working week that the surveyee $\theta$ plans to take between $e_{w}$ and $e_{w}^{\prime}$ (in any direction) during the hours of operation of the rapid transit network.

Let $W_{\theta}=\left\{w \in W \mid \alpha_{w}(\theta)>0\right\} \quad \forall \theta \in \Theta$ (notice that $W_{\theta}$ is the set of distinct pairs of key station locations between which the surveyee $\theta$ plans to travel, without taking into consideration the direction of the related trips).

For each $w \in W$, let

$$
\widehat{t}_{w}=\bar{t}\left(e_{w}\right)+\frac{\bar{t}_{a}}{2}+\frac{60}{\bar{v}} d_{e_{w} e_{w}^{\prime}}+\bar{t}\left(e_{w}^{\prime}\right)
$$

where $d_{e_{w} e_{w}^{\prime}}$ is the Euclidean distance (expressed in kilometers) between $e_{w}$ and $e_{w}^{\prime}$ if $\left\{e_{w}, e_{w}^{\prime}\right\} \notin E$. We are considering $\widehat{t}_{w}$ as the minimum possible average time that the trip between $e_{w}$ and $e_{w}^{\prime}$ (in any direction) will take on the rapid transit network (this minimum will be reached if $\left\{e_{w}, e_{w}^{\prime}\right\} \in E, s_{e_{w} e_{w}^{\prime}}=0$ and link $\left\{e_{w}, e_{w}^{\prime}\right\}$ is selected to be constructed).

For each $\theta \in \Theta$ and for each $w \in W_{\theta}$, let $\tau_{w}(\theta)$ be the maximum amount of time (expressed in minutes) that the surveyee $\theta$ is willing to spend for traveling between $e_{w}$ and $e_{w}^{\prime}$ (in any direction). Without loss of generality it will be assumed that $\tau_{w}(\theta) \geqslant \widehat{t}_{w}$ (if $\tau_{w}(\theta)<\widehat{t}_{w}$, then the surveyee $\theta$ will not utilize the rapid transit network for traveling between $e_{w}$ 
and $e_{w}^{\prime}$, and, consequently, we shall set $\alpha_{w}(\theta)=0$, hence $w \notin W_{\theta}$ and the value of $\tau_{w}(\theta)$ will not be considered).

In order to determine an initial setting for the headways in a subsequent stage, the surveyees could also be asked about the starting time and the direction of their trips.

Let $W^{\prime}=\bigcup_{\theta \in \Theta} W_{\theta}$ (notice that $W^{\prime}$ is the set of distinct pairs of key station locations between which the surveyees plan to travel, without taking into consideration the direction of the related trips). It is expected that $W^{\prime}=W$, since the cardinality of $\Theta$ should be large enough for the survey results to be reliable.

For each $w \in W^{\prime}$, let $\Theta_{w}=\left\{\theta \in \Theta \mid \alpha_{w}(\theta)>\right.$ $0\}$ (notice that $\Theta_{w}$ is the set of surveyees that plan to travel between $e_{w}$ and $e_{w}^{\prime}$, without taking into consideration the direction of the related trips). In order to estimate the number of weekly trips that the surveyees in $\Theta_{w}$ will take on the rapid transit network between $e_{w}$ and $e_{w}^{\prime}$ (in any direction), we group the values $\left\{\tau_{w}(\theta)\right\}_{\theta \in \Theta_{w}}$ into $q(w)$ time intervals $\left[u_{w}^{1}, u_{w}^{2}\right), \ldots,\left[u_{w}^{q(w)-1}, u_{w}^{q(w)}\right)$, $\left[u_{w}^{q(w)},+\infty\right)$, where $q(w) \in \mathbb{N}, u_{w}^{1}=\min \left\{\tau_{w}(\theta) \mid \theta \in\right.$ $\left.\Theta_{w}\right\}, u_{w}^{2}, \ldots, u_{w}^{q(w)} \in \mathbb{R}$ and $u_{w}^{1}<u_{w}^{2}<\ldots<u_{w}^{q(w)} \leqslant$ $\max \left\{\tau_{w}(\theta) \mid \theta \in \Theta_{w}\right\}$, and we define

$$
g_{w}^{k}=\sum_{\theta \in \Theta_{w}^{k}} \alpha_{w}(\theta) \quad \forall k \in\{1, \ldots, q(w)\},
$$

where $\Theta_{w}^{k}=\left\{\theta \in \Theta_{w} \mid \tau_{w}(\theta) \geqslant u_{w}^{k}\right\}$ (the value of $g_{w}^{k}$ can be interpreted as the expected number of weekly trips taken on the rapid transit network by the surveyees that plan to travel between $e_{w}$ and $e_{w}^{\prime}$, assuming that the fastest route for taking them takes $u_{w}^{k}$ minutes, as well as that the capacity of the rapid transit network is enough to hold all those trips). It will also be assumed that the values of $q(w)$ and $\left\{u_{w}^{k}\right\}_{k \in\{2, \ldots, q(w)\}}$ have been set in such a way that $g_{w}^{1}>g_{w}^{2}>\ldots>g_{w}^{q(w)}$ (notice that $g_{w}^{q(w)} \geqslant 1$ ). Algorithm 1 in Section 4 provides one possible method for setting the values of $q(w)$ and $\left\{u_{w}^{k}\right\}_{k \in\{2, \ldots, q(w)\}}$ in such a way that the above assumptions are satisfied, although some other method could be utilized.

\section{Station and Link Location}

In this section we present an integer linear optimization model for selecting the stations to be constructed and the links between them, so that the resulting rapid transit network is connected and its construction cost does not exceed the available budget. This model is based on the one proposed in Escudero and Muñoz ${ }^{13}$, for the particular case where at least one key station has to be constructed at each location; see also Escudero and Muñoz ${ }^{12}$.

We attach more importance to linking two station locations by one line, than not linking them in exchange of linking some two other station locations by more than one line. Thus, in this first stage of the approach we implicitly assume that whichever two station locations are linked by one line at most, whereas in the second stage we shall check whether, without eliminating the already selected links, it is possible and advisable to have pairs of station locations linked by more than one line (see Sections 6, 7 and 8 of Escudero and Muñoz ${ }^{15}$ ).

Each feasible solution to the model will define a route for traveling between each pair of key station locations. These routes are understood as preliminary routes for taking the trips on the rapid transit network (it will be attempted to improve them in the second stage of the approach; see Section 5 of Escudero and Muñoz ${ }^{15}$ ).

The optimization criterion is the maximization of an estimation of the number of weekly trips that the surveyees will take on the rapid transit network, which is equivalent to maximizing an estimation of the gross profits, assuming that the users have to buy a ticket per trip and that there is a unique fare for the tickets. In order to do this estimation, we consider the average time for the preliminary routes for taking the trips demanded by the surveyees (since the lines have not still been defined at this stage, these average times are calculated assuming that no transfers are required).

We define the following variables (the definition of these variables and the rest of notation below can 
be found listed and summarized in Appendix B):

$$
\begin{aligned}
& x_{i j}=\left\{\begin{array}{ll}
1 & \text { if } i \text { and } j \text { are linked } \\
0 & \text { otherwise }
\end{array} \forall\{i, j\} \in E\right. \\
& \gamma_{i}= \begin{cases}1 & \text { if } \sum_{j \in \Gamma(i), j>i} x_{i j}+\sum_{j \in \Gamma(i), j<i} x_{j i} \text { is odd } \\
0 & \text { otherwise }\end{cases} \\
& \forall i \in V
\end{aligned}
$$$$
\Delta_{i} \in\{0, \ldots, r(i)\} \quad \forall i \in V
$$

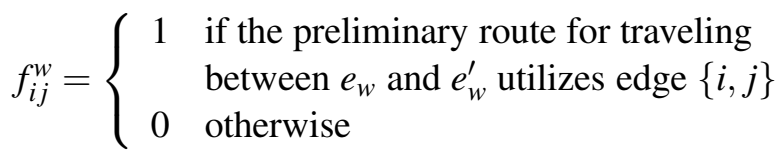
$\forall w \in W, \forall\{i, j\} \in E$

$\varepsilon_{i}^{w}= \begin{cases}1 & \begin{array}{l}\text { if the preliminary route for traveling } \\ \text { between } e_{w} \text { and } e_{w}^{\prime} \text { passes through } i\end{array} \\ 0 & \text { otherwise }\end{cases}$ $\forall w \in W, \forall i \in V \backslash\left\{e_{w}, e_{w}^{\prime}\right\}$

$p_{w}^{k} \in\{0,1\} \quad \forall w \in W^{\prime}, \forall k \in\{1, \ldots, q(w)\}$,

where

$$
\begin{aligned}
r(i) & = \begin{cases}\frac{|\Gamma(i)|}{2} & \text { if }|\Gamma(i)| \text { is even, } \\
\frac{|\Gamma(i)|-1}{2} & \text { if }|\Gamma(i)| \text { is odd, }\end{cases} \\
\Delta_{i} & =\frac{\sum_{j \in \Gamma(i), j>i} x_{i j}+\sum_{j \in \Gamma(i), j<i} x_{j i}-\gamma_{i}}{2},
\end{aligned}
$$

and a necessary condition for $p_{w}^{k}$ to take the value 1 is that the average time for traveling between $e_{w}$ and $e_{w}^{\prime}$ by following the associated preliminary route is less or equal to $u_{w}^{k}$.

We propose the following model:

$$
\begin{gathered}
\text { Maximize } z=\sum_{w \in W^{\prime}} \sum_{k=1}^{q(w)} g_{w}^{k} p_{w}^{k} \\
\text { subject to: } \\
\sum_{j \in \Gamma(i), j>i} x_{i j}+\sum_{j \in \Gamma(i), j<i} x_{j i}=2 \Delta_{i}+\gamma_{i} \quad \forall i \in V \\
\sum_{i \in V} a_{i}\left(\Delta_{i}+\gamma_{i}\right)+\sum_{\{i, j\} \in E} c_{i j} x_{i j} \leqslant b \\
f_{i j}^{w} \leqslant x_{i j} \quad \forall w \in W, \forall\{i, j\} \in E
\end{gathered}
$$

$$
\begin{array}{r}
\sum_{\substack{j \in \Gamma(i) \\
j>i}} f_{i j}^{w}+\sum_{\substack{j \in \Gamma(i) \\
j<i}} f_{j i}^{w}= \begin{cases}1 & \text { if } i \in\left\{e_{w}, e_{w}^{\prime}\right. \\
2 \varepsilon_{i}^{w} & \text { otherwise }\end{cases} \\
\forall w \in W, \forall i \in V
\end{array}
$$

$$
\begin{array}{r}
\bar{t}^{\prime}(w)+\sum_{\{i, j\} \in E} \bar{t}_{i j}^{\prime} f_{i j}^{w}-u_{w}^{k} \leqslant M_{w}^{k}\left(1-p_{w}^{k}\right) \\
\forall w \in W^{\prime}, \forall k \in\{1, \ldots, q(w)\}
\end{array}
$$

$$
\begin{gathered}
x_{i j} \in\{0,1\} \quad \forall\{i, j\} \in E \\
\gamma_{i} \in\{0,1\} \quad \forall i \in V
\end{gathered}
$$$$
\Delta_{i} \in\{0, \ldots, r(i)\} \quad \forall i \in V
$$

$$
f_{i j}^{w} \in\{0,1\} \quad \forall w \in W, \forall\{i, j\} \in E
$$

$$
\begin{gathered}
\varepsilon_{i}^{w} \in\{0,1\} \quad \forall w \in W, \forall i \in V \backslash\left\{e_{w}, e_{w}^{\prime}\right\} \\
p_{w}^{k} \in\{0,1\} \quad \forall w \in W^{\prime}, \forall k \in\{1, \ldots, q(w)\},
\end{gathered}
$$

where $\bar{t}^{\prime}(w)=\bar{t}\left(e_{w}\right)+\frac{\bar{t}_{a}}{2}-\bar{t}_{s}+\bar{t}\left(e_{w}^{\prime}\right), \bar{t}_{i j}^{\prime}=\frac{60}{\bar{v}} d_{i j}+$ $\bar{t}_{s}\left(s_{i j}+1\right)$, and $M_{w}^{k}$ is an upper bound for the value of $\bar{t}^{\prime}(w)+\sum_{\{i, j\} \in E} \bar{t}_{i j}^{\prime} f_{i j}^{w}-u_{w}^{k}$ over the feasible region of the above model relaxation resulting from eliminating the constraints (5) and (6), and the variables $\left\{p_{w}^{k}\right\}_{w \in W^{\prime}, k \in\{1, \ldots, q(w)\}}$.

Constraints (1) and (2) impose the budget constraint. Constraints (3) and (4) define the preliminary routes and guarantee that any feasible solution to this model will give rise to a connected rapid transit network. Constraints (5) impose that, for each $w \in W^{\prime}$, at most one of the variables $\left\{p_{w}^{k}\right\}_{k \in\{1, \ldots, q(w)\}}$ can be equal to 1 . Constraints (6) impose that, for each $w \in W^{\prime}$ and for each $k \in\{1, \ldots, q(w)\}$, if $p_{w}^{k}=1$ then $\bar{t}^{\prime}(w)+\sum_{\{i, j\} \in E} \bar{t}_{i j}^{\prime} f_{i j}^{w} \leqslant u_{w}^{q}$ (we are considering $\bar{t}^{\prime}(w)+\sum_{\{i, j\} \in E} \bar{t}_{i j}^{\prime} f_{i j}^{w}$ as the average time for traveling between $e_{w}$ and $e_{w}^{\prime}$ by following the associated preliminary route, since $\sum_{\{i, j\} \in E} \bar{t}_{i j}^{\prime} f_{i j}^{w}-\bar{t}_{s}$ is the average time for traveling between the boarding and alighting platforms at $e_{w}$ and $e_{w}^{\prime}$ for this preliminary route, assuming that no transfers are required). 
Given that any preliminary route for traveling between two key station locations will utilize at most $n-1$ edges in $E$, it is possible to take $M_{w}^{k}=\bar{t}^{\prime}(w)+$ $\bar{t}_{n-1}^{\prime}-u_{w}^{k} \quad \forall w \in W^{\prime}, \forall k \in\{1, \ldots, q(w)\}$, where $\bar{t}_{n-1}^{\prime}$ is the sum of the $n-1$ greatest values in $\left\{\bar{t}_{i j}^{\prime}\right\}_{\{i, j\} \in E}$.

For each $w \in W^{\prime}$, in the objective function we are considering $\sum_{k=1}^{q(w)} g_{w}^{k} p_{w}^{k}$ as the estimation for the number of weekly trips taken on the rapid transit network by the surveyees that plan to travel between $e_{w}$ and $e_{w}^{\prime}$. Since we are dealing with a maximization problem, each optimal solution to the above model will satisfy the following properties (the first one will also be satisfied by any feasible solution to the model):

(i) For each $w \in W^{\prime}$ such that

$$
\bar{t}^{\prime}(w)+\sum_{\{i, j\} \in E} \bar{t}_{i j}^{\prime} f_{i j}^{w}>u_{w}^{k} \quad \forall k \in\{1, \ldots, q(w)\},
$$

it will be $p_{w}^{k}=0 \quad \forall k \in\{1, \ldots, q(w)\}$.

(ii) For each $w \in W^{\prime}$ such that $\exists k \in\{1, \ldots, q(w)\}$ with

$$
\bar{t}^{\prime}(w)+\sum_{\{i, j\} \in E} \bar{t}_{i j}^{\prime} f_{i j}^{w} \leqslant u_{w}^{k},
$$

it will be $p_{w}^{k^{*}}=1$ and $p_{w}^{k}=0 \quad \forall k \in$ $\{1, \ldots, q(w)\} \backslash\left\{k^{*}\right\}$, where

$$
\begin{aligned}
& k^{*}=\min \{k \in\{1, \ldots, q(w)\} \mid \\
& \left.\bar{t}^{\prime}(w)+\sum_{\{i, j\} \in E} \bar{t}_{i j}^{\prime} f_{i j}^{w} \leqslant u_{w}^{k}\right\} .
\end{aligned}
$$

For the particular case where $q(w)=1 \quad \forall w \in$ $W^{\prime}$, the above objective function is the same as in the model from Escudero and Muñoz ${ }^{13}$, i.e., the allor-nothing model for the potential users' behavior from Marín ${ }^{8}$ is considered.

The greater the values of $\{q(w)\}_{w \in W^{\prime}}$, the more accurate the estimation of the number of weekly trips that the surveyees will take on the rapid transit network (assuming that no transfers are required) but also the more variables and constraints in the above model.

We have considered some other objective functions that do not require either the constraints (5) and
(6) or the variables $\left\{p_{w}^{k}\right\}_{w \in W^{\prime}, k \in\{1, \ldots, q(w)\}}$, but the global computational results came out worse than for the above objective function.

In order to illustrate the application of the first stage of our proposed approach, a small-size instance is provided in the following example:

Example 1. Consider the graph $G=$ $(V, E)$, where $V=\{1,2,3,4,5,6\}$ and $E=$ $\{\{1,2\},\{1,4\},\{1,5\},\{2,3\},\{2,6\},\{4,5\},\{5,6\}\}$ (see Figure 1). Then $\Gamma(1)=\{2,4,5\}$, $\Gamma(2)=\{1,3,6\}, \quad \Gamma(3)=\{2\}, \quad \Gamma(4)=\{1,5\}$, $\Gamma(5)=\{1,4,6\}, \quad \Gamma(6)=\{2,5\} \quad$ and $W=$ $\{(1,2),(1,3),(1,4),(1,5),(1,6),(2,3),(2,4),(2,5)$, $(2,6),(3,4),(3,5),(3,6),(4,5),(4,6),(5,6)\}$.

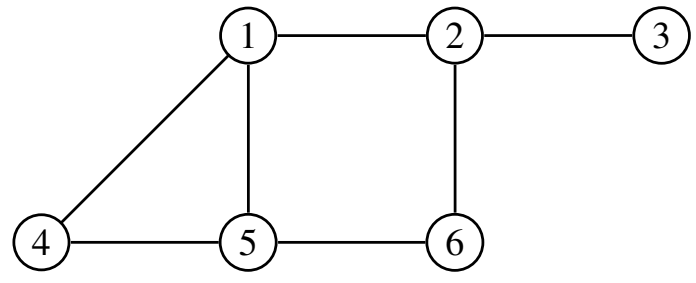

Fig. 1. Graphic representation of $G=(V, E)$

Let $a_{i}=30 \quad \forall i \in V, d_{12}=1.6, d_{14}=2.1, d_{15}=$ $1, d_{23}=1.1, d_{26}=1.5, d_{45}=1.2, d_{56}=0.9, s_{i j}=$ $0 \quad \forall\{i, j\} \in E, c_{i j}=45 d_{i j} \quad \forall\{i, j\} \in E, b=645$, $\bar{v}=60, \bar{t}(i)=2 \quad \forall i \in V, \bar{t}_{a}=4, \bar{t}_{s}=0.5$ and $\bar{t}_{r}=3$ $\left(\left\{a_{i}\right\}_{i \in V},\left\{c_{i j}\right\}_{\{i, j\} \in E}\right.$ and $b$ are expressed in millions of euros). Then $\bar{t}_{i j}^{\prime}=d_{i j}+0.5 \quad \forall\{i, j\} \in E$.

Let $\Theta=\left\{\theta_{1}, \ldots, \theta_{5}\right\}, W_{\theta_{1}}=\{(1,2),(1,6),(2,4)$, $(2,6)\}, \quad W_{\theta_{2}}=\{(1,4),(1,5),(4,5)\}, \quad W_{\theta_{3}}=$ $\{(1,6),(2,5),(3,5)\}, \quad W_{\theta_{4}}=\{(1,3),(1,6),(2,3)$, $(3,5),(3,6),(5,6)\}$ and $W_{\theta_{5}}=\{(1,6),(2,4),(3,4)$, $(3,6),(4,6)\}$. Therefore, we have $W^{\prime}=W$ and $\bar{t}^{\prime}(w)=5.5 \quad \forall w \in W^{\prime}$.

For each $\theta \in \Theta$ and for each $w \in W_{\theta}$, Table 1 shows the values we have considered for $\alpha_{w}(\theta)$ and $\tau_{w}(\theta)$. 
Table 1. Values of $\left\{\alpha_{w}(\theta)\right\}_{\theta \in \Theta, w \in W_{\theta}}$ and $\left\{\tau_{w}(\theta)\right\}_{\theta \in \Theta, w \in W_{\theta}}$

\begin{tabular}{|c|c|c|c|}
\hline$\theta$ & $w$ & $\alpha_{w}(\theta)$ & $\tau_{w}(\theta)$ \\
\hline \multirow[t]{4}{*}{$\theta_{1}$} & $(1,2)$ & 1 & 8 \\
\hline & $(1,6)$ & 2 & 12 \\
\hline & $(2,4)$ & 5 & 10 \\
\hline & $(2,6)$ & 1 & 9 \\
\hline \multirow[t]{3}{*}{$\theta_{2}$} & $(1,4)$ & 1 & 10 \\
\hline & $(1,5)$ & 1 & 7 \\
\hline & $(4,5)$ & 1 & 8 \\
\hline \multirow[t]{3}{*}{$\theta_{3}$} & $(1,6)$ & 1 & 13 \\
\hline & $(2,5)$ & 1 & 9 \\
\hline & $(3,5)$ & 8 & 10 \\
\hline \multirow[t]{6}{*}{$\theta_{4}$} & $(1,3)$ & 1 & 12 \\
\hline & $(1,6)$ & 10 & 8 \\
\hline & $(2,3)$ & 1 & 8 \\
\hline & $(3,5)$ & 1 & 12 \\
\hline & $(3,6)$ & 1 & 16 \\
\hline & $(5,6)$ & 1 & 8 \\
\hline \multirow[t]{5}{*}{$\theta_{5}$} & $(1,6)$ & 5 & 9 \\
\hline & $(2,4)$ & 1 & 12 \\
\hline & $(3,4)$ & 1 & 15 \\
\hline & $(3,6)$ & 12 & 9 \\
\hline & $(4,6)$ & 1 & 9 \\
\hline
\end{tabular}

For each $w \in W^{\prime}$, Table 2 shows the values we have considered for $q(w),\left\{u_{w}^{k}\right\}_{k \in\{1, \ldots, q(w)\}}$, $\left\{g_{w}^{k}\right\}_{k \in\{1, \ldots, q(w)\}}$ and $\left\{M_{w}^{k}\right\}_{k \in\{1, \ldots, q(w)\}}$ (the values of $q(w),\left\{u_{w}^{k}\right\}_{k \in\{2, \ldots, q(w)\}}$ and $\left\{g_{w}^{k}\right\}_{k \in\{1, \ldots, q(w)\}}$ have been computed by applying Algorithm 1 in Section 4 taking $\bar{q}=2$, and we have taken $M_{w}^{k}=\bar{t}^{\prime}(w)+$ $\left.\bar{t}_{n-1}^{\prime}-u_{w}^{k} \quad \forall k \in\{1, \ldots, q(w)\}\right)$.
Table 2. Values of $\{q(w)\}_{w \in W^{\prime}}, \quad\left\{u_{w}^{k}\right\}_{w \in W^{\prime}, k \in\{1, \ldots, q(w)\}}$, $\left\{g_{w}^{k}\right\}_{w \in W^{\prime}, k \in\{1, \ldots, q(w)\}}$ and $\left\{M_{w}^{k}\right\}_{w \in W^{\prime}, k \in\{1, \ldots, q(w)\}}$

\begin{tabular}{ccccc}
\hline$w$ & $q(w)$ & $\begin{array}{c}\left\{u_{w}^{k}\right\} \\
k \in\{1, \ldots, q(w)\}\end{array}$ & $\begin{array}{c}\left\{g_{w}^{k}\right\} \\
k \in\{1, \ldots, q(w)\}\end{array}$ & $\begin{array}{c}\left\{M_{w}^{k}\right\} \\
k \in\{1, \ldots, q(w)\}\end{array}$ \\
\hline$(1,2)$ & 1 & 8 & 1 & 7.5 \\
$(1,3)$ & 1 & 12 & 1 & 3.5 \\
$(1,4)$ & 1 & 10 & 1 & 5.5 \\
$(1,5)$ & 1 & 7 & 1 & 8.5 \\
$(1,6)$ & 2 & 8,9 & 18,8 & $7.5,6.5$ \\
$(2,3)$ & 1 & 8 & 1 & 7.5 \\
$(2,4)$ & 1 & 10 & 6 & 5.5 \\
$(2,5)$ & 1 & 9 & 1 & 6.5 \\
$(2,6)$ & 1 & 9 & 1 & 6.5 \\
$(3,4)$ & 1 & 15 & 1 & 0.5 \\
$(3,5)$ & 1 & 10 & 9 & 5.5 \\
$(3,6)$ & 2 & 9,16 & 13,1 & $6.5,-0.5$ \\
$(4,5)$ & 1 & 8 & 1 & 7.5 \\
$(4,6)$ & 1 & 9 & 1 & 6.5 \\
$(5,6)$ & 1 & 8 & 1 & 7.5 \\
\hline
\end{tabular}

Upon solving the above model for this particular instance, we obtain that all links except $\{1,4\}$ are constructed, the construction cost of the rapid transit network is 568.5 million euros, and the optimal value of the objective function is 20 .

\section{Computational Experience}

We have randomly generated five example cases $C 1, \ldots, C 5$ by using the following procedure $(C 1$, $C 2$ and $C 3$ were also considered in Escudero and Muñoz ${ }^{15}$ ):

The $n$ key station locations have been randomly generated from a continuous uniform distribution on a square of given side length $\rho$ (expressed in kilometers), in such a way that whichever two of them are at least two kilometers apart (considering the Euclidean distance). The pairs of key station locations that can potentially be linked are the ones with the $m$ shortest Euclidean distances between them.

We have set the parameter values having a metro system in mind.

For each example case, Table 3 shows the values we have taken for $\rho, n, m, b$ and $|\Theta|$ ( $b$ is expressed in millions of euros). 
Table 3. Values of $\rho, n, m, b$ and $|\Theta|$ for the example cases

\begin{tabular}{cccccc}
\hline & $\rho$ & $n$ & $m$ & $b$ & $|\Theta|$ \\
\hline$C 1$ & 10 & 15 & 35 & 6000 & 1500 \\
$C 2$ & 10 & 20 & 45 & 8000 & 2000 \\
$C 3$ & 15 & 25 & 60 & 13000 & 3000 \\
$C 4$ & 15 & 30 & 75 & 16000 & 3500 \\
$C 5$ & 15 & 35 & 90 & 18000 & 4000 \\
\hline
\end{tabular}

For all the example cases we have set the values for the rest of the parameters as follows: $a_{i}=$ $30 \forall i \in V$ (expressed in millions of euros), $d_{i j}$ is the Euclidean distance between $i$ and $j \quad \forall(i, j) \in W$ (expressed in kilometers), $s_{i j}=\left[d_{i j}\right]-1 \quad \forall\{i, j\} \in$ $E, c_{i j}=45 d_{i j}+30 s_{i j} \quad \forall\{i, j\} \in E$ (expressed in millions of euros), $\bar{v}=55, \bar{t}(i)=3 \quad \forall i \in V, \bar{t}_{a}=5$, $\bar{t}_{s}=0.6$ and $\bar{t}_{r}=4$.

Figures 2-6 show the underlying graphs of $C 1, \ldots, C 5$, respectively.

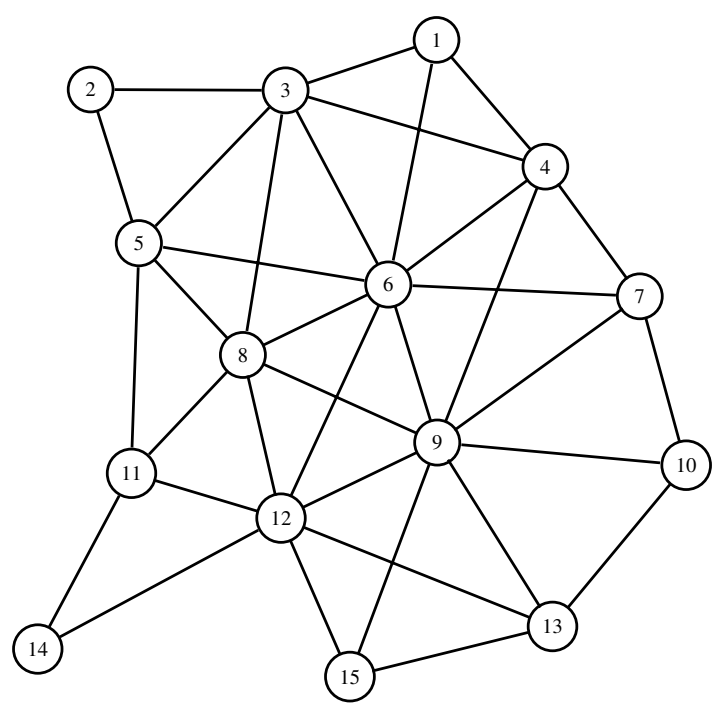

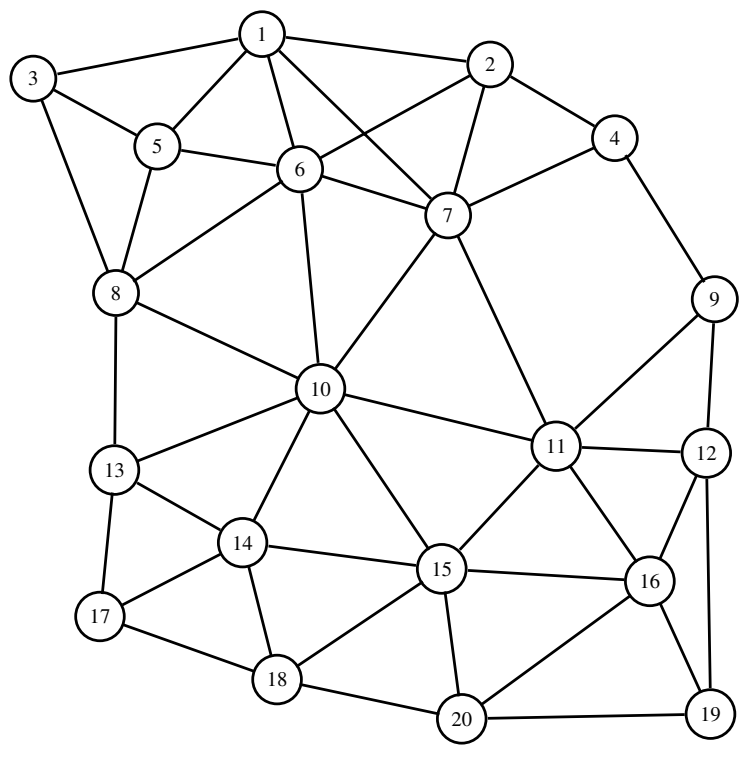

Fig. 3. Graphic representation of the underlying graph of C2

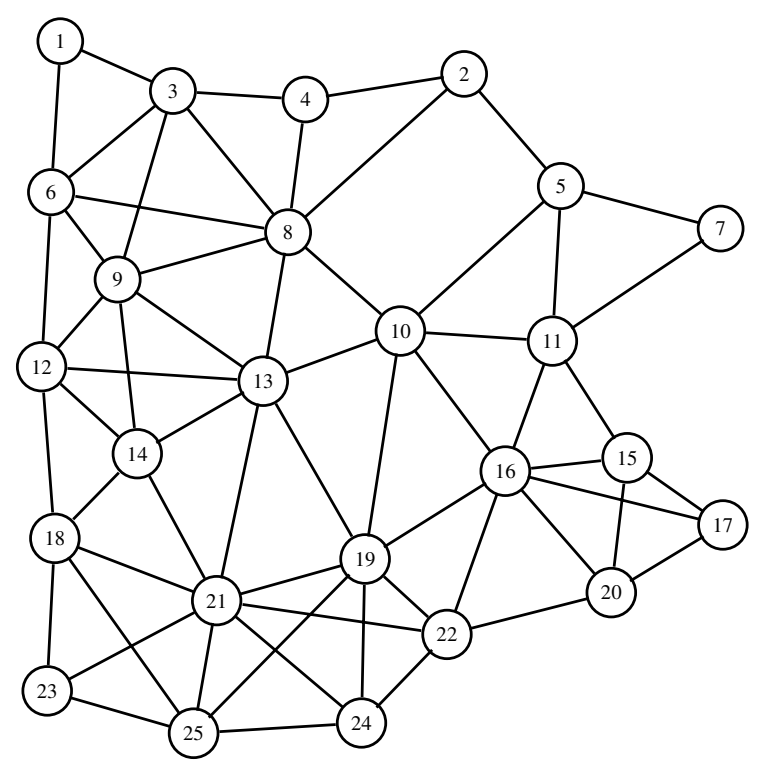

Fig. 4. Graphic representation of the underlying graph of C3

Fig. 2. Graphic representation of the underlying graph of C1 


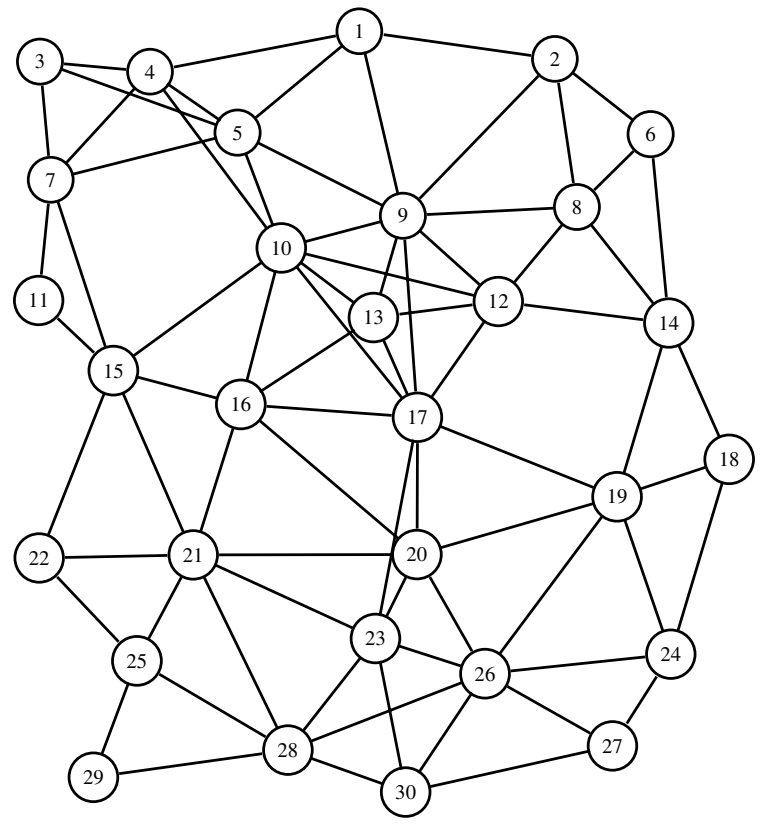

Fig. 5. Graphic representation of the underlying graph of C4

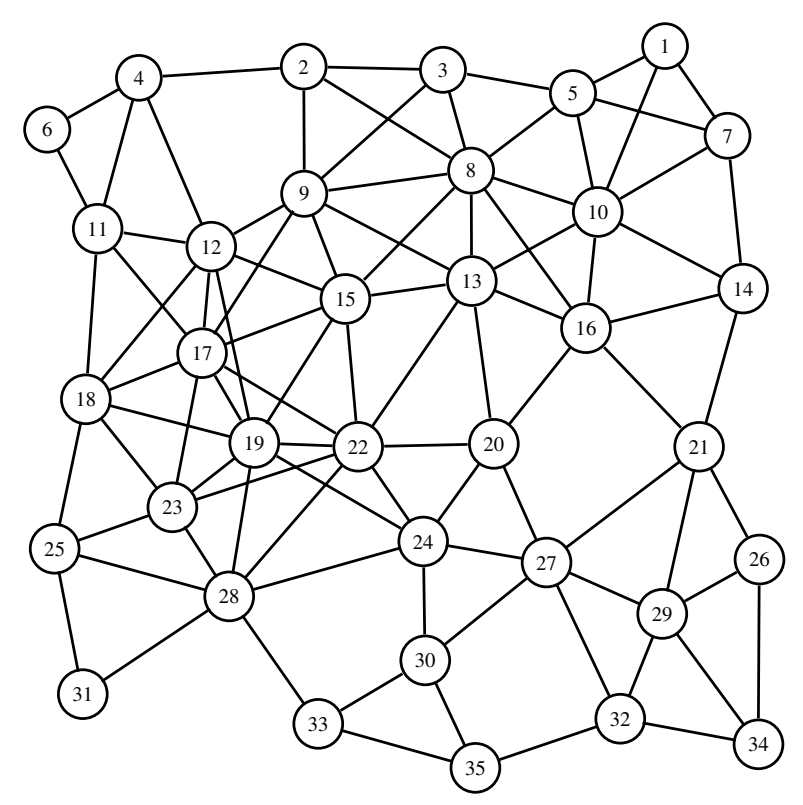

Fig. 6. Graphic representation of the underlying graph of C5

For each $\theta \in \Theta$, the procedure we have utilized for generating the survey answers $\left\{\alpha_{w}(\theta)\right\}_{w \in W}$ and $\left\{\tau_{w}(\theta)\right\}_{w \in W_{\theta}}$ for the surveyee $\theta$ is as follows:

1. We set the number $\beta_{\theta}$ of distinct trips that the surveyee $\theta$ plans to take on the rapid transit network, without taking into consideration their direction, imposing that $\beta_{\theta} \in\{1, \ldots, 5\}$ (notice that $\beta_{\theta}=\left|W_{\theta}\right|$ ). For this purpose, we generate the value of $\beta_{\theta}$ in such a way that $P\left(\beta_{\theta}=k\right)=0.3 \quad \forall k \in\{1,2\}, P\left(\beta_{\theta}=3\right)=$ 0.2 and $P\left(\beta_{\theta}=k\right)=0.1 \quad \forall k \in\{4,5\}$.

2. We set the $\beta_{\theta}$ distinct pairs of key station locations $w_{1}(\theta), \ldots, w_{\beta_{\theta}}(\theta)$ between which the surveyee $\theta$ plans to travel from the $\frac{n(n-1)}{2}$ pairs in $W$ (notice that $W_{\theta}=$ $\left.\left\{w_{1}(\theta), \ldots, w_{\beta_{\theta}}(\theta)\right\}\right)$. For this purpose, let us denote $W=\left\{w_{1}, \ldots, w_{\frac{n(n-1)}{2}}\right\}$. For each $k \in\left\{1, \ldots, \beta_{\theta}\right\}$, we randomly generate a value $\varphi_{k}$ from a discrete uniform distribution on $\left\{1, \ldots, \frac{n(n-1)}{2}\right\} \backslash \bigcup_{k^{\prime}=1}^{k-1}\left\{\varphi_{k^{\prime}}\right\}$ and set $w_{k}(\theta)=$ $w_{\varphi_{k}}$.

3. We set the number $\eta_{\theta}$ of weekly trips that the surveyee $\theta$ plans to take on the rapid transit network, imposing that $\eta_{\theta} \in\left\{\beta_{\theta}, \ldots, 21\right\}$ (notice that $\eta_{\theta}=\sum_{w \in W_{\theta}} \alpha_{w}(\theta)$ ). For this purpose, we generate the value of $\eta_{\theta}$ in such a way that $P\left(\eta_{\theta}=k\right)=\frac{1}{2\left(17-\beta_{\theta}\right)} \quad \forall k \in$ $\left\{\beta_{\theta}, \ldots, 9\right\} \cup\{15, \ldots, 21\}$ and $P\left(\eta_{\theta}=k\right)=$ $0.1 \quad \forall k \in\{10, \ldots, 14\}$.

4. We set the values of $\left\{\alpha_{w}(\theta)\right\}_{w \in W}$ imposing that $\sum_{w \in W_{\theta}} \alpha_{w}(\theta)=\eta_{\theta}$. For this purpose, for each $k \in\left\{1, \ldots, \beta_{\theta}-1\right\}$ we generate the value of $\alpha_{w_{k}(\theta)}(\theta)$ from a discrete uniform distribution on $\left\{1, \ldots, \eta_{\theta}-\sum_{k^{\prime}=1}^{k-1} \alpha_{w_{k^{\prime}}(\theta)}(\theta)-\beta_{\theta}+k\right\}$ and set $\alpha_{w_{\beta_{\theta}}(\theta)}(\theta)=\eta_{\theta}-\sum_{k^{\prime}=1}^{\beta_{\theta}-1} \alpha_{w_{k^{\prime}}(\theta)}(\theta)$ and $\alpha_{w}(\theta)=0 \quad \forall w \in W \backslash W_{\theta}$.

5. We set the values of $\left\{\tau_{w}(\theta)\right\}_{w \in W_{\theta}}$ imposing that $\tau_{w}(\theta) \in\left\{\left[\widehat{t}_{w}\right\rceil, \ldots,\left\lceil\widehat{t}_{w}\left(1+\frac{3}{2} \sqrt{\frac{\bar{d}}{d_{e_{w} e_{w}^{\prime}}}}\right)\right\rceil\right\}$ $\forall w \in W_{\theta}$, where $\bar{d}=\max \left\{d_{e_{w} e_{w}^{\prime}} \mid w \in W\right\}$ (notice that, without loss of generality, in Section 2 it was assumed that $\tau_{w}(\boldsymbol{\theta}) \geqslant$ $\left.\widehat{t}_{w} \quad \forall w \in W_{\theta}\right)$. For this purpose, for each $w \in W_{\theta}$ we generate the value of $\tau_{w}(\theta)$ 


$$
\begin{aligned}
& \text { from a discrete uniform distribution on } \\
& \left\{\left\lceil\widehat{t}_{w}\right\rceil, \ldots,\left\lceil\widehat{t}_{w}\left(1+\frac{3}{2} \sqrt{\frac{\bar{d}}{d_{e_{w} e_{w}^{\prime}}}}\right)\right\rceil\right\} .
\end{aligned}
$$

It is worth noting that it is not relevant to know explicitly the specific pairs of key stations locations between which each surveyee plans to travel, since, for each $w \in W^{\prime}$, the values $\left\{\alpha_{w}(\theta)\right\}_{\theta \in \Theta_{w}}$ and $\left\{\tau_{w}(\theta)\right\}_{\theta \in \Theta_{w}}$ are actually treated as the set $\left\{\left(\alpha_{w}(\theta), \tau_{w}(\theta)\right)\right\}_{\theta \in \Theta_{w}}$, which can be considered simply as a set of 2-dimensional vectors independent of the surveyees. Thus, when breaking down the survey results, the surveyees are indistinguishable.

For all the example cases it has been obtained that $W^{\prime}=W$.

Let us impose an upper bound $\bar{q} \in$ $\left\{1, \ldots, \max \left\{g_{w}^{1} \mid w \in W^{\prime}\right\}\right\}$ on the values of $\{q(w)\}_{w \in W^{\prime}}$ (notice that $g_{w}^{1}=\sum_{\theta \in \Theta_{w}} \alpha_{w}(\theta) \quad \forall w \in$ $W^{\prime}$, since $u_{w}^{1}=\min \left\{\tau_{w}(\theta) \mid \theta \in \Theta_{w}\right\}$ by definition, and, hence, $\left.\Theta_{w}^{1}=\Theta_{w}\right)$, and let $\mu=\frac{\max \left\{g_{w}^{1} \mid w \in W^{\prime}\right\}}{\bar{q}}$ (notice that $\mu \geqslant 1$ ).

Let $w \in W^{\prime}$. Algorithm 1 below provides a simple method for setting the values of $q(w)$ and $\left\{u_{w}^{k}\right\}_{k \in\{2, \ldots, q(w)\}}$ by making use of $\mu$, in such a way that the assumptions stated in Section 2 are satisfied. It also computes the values of $\left\{g_{w}^{k}\right\}_{k \in\{1, \ldots, q(w)\}}$.

Let $o_{w}=\left|\Theta_{w}\right|$. For simplicity of notation, without loss of generality we denote $\Theta_{w}$ by $\left\{\theta_{1}, \ldots, \theta_{o_{w}}\right\}$, where $\tau_{w}\left(\theta_{1}\right) \leqslant \ldots \leqslant \tau_{w}\left(\theta_{o_{w}}\right)$ (notice that $u_{w}^{1}=$ $\left.\tau_{w}\left(\theta_{1}\right)\right)$.

The output values of Algorithm 1 will satisfy that $\forall k \in\{2, \ldots, q(w)\} \exists j(k) \in\left\{2, \ldots, o_{w}\right\}$ such that $u_{w}^{k}=\tau_{w}\left(\theta_{j(k)}\right)$, where $j(k)>j(k-1), \tau_{w}\left(\theta_{j(k)-1}\right)<$ $\tau_{w}\left(\theta_{j(k)}\right)$ and $g_{w}^{k-1}-g_{w}^{k} \geqslant \mu$. Therefore, it will be $g_{w}^{k-1}-g_{w}^{k}=\sum_{j^{\prime}=j(k-1)}^{j(k)-1} \alpha_{w}\left(\theta_{j^{\prime}}\right) \quad \forall k \in\{2, \ldots, q(w)\}$.

For each $k \in\{1, \ldots, q(w)-1\}$, Algorithm 1 decides whether to either set $q(w)=k$ and stop, or to set $k=k+1$ and compute the values of $j(k), u_{w}^{k}$ and $g_{w}^{k}$. In order to make such decision, it implicitly checks whether the set $U_{k}=\left\{j \in\left\{j(k)+1, \ldots, o_{w}\right\} \mid\right.$ $\left.\tau_{w}\left(\theta_{j-1}\right)<\tau_{w}\left(\theta_{j}\right), \sum_{j^{\prime}=j(k)}^{j-1} \alpha_{w}\left(\theta_{j^{\prime}}\right) \geqslant \mu\right\}$ is empty or not. If it is empty, it sets $q(w)=k$ and stops; if not, it sets $k=k+1, j(k)=\min \left\{j \mid j \in U_{k-1}\right\}$, $u_{w}^{k}=\tau_{w}\left(\theta_{j(k)}\right)$ and $g_{w}^{k}=g_{w}^{k-1}-\sum_{j^{\prime}=j(k-1)}^{j(k)-1} \alpha_{w}\left(\theta_{j^{\prime}}\right)$.
At each $k$ th iteration, the index $j(k)$ is denoted by $i$, and the index $j(k+1)$ to be set is denoted by $j+1$ (see Steps 1 and 5). Instead of explicitly determining the set $U_{k}$, Algorithm 1 proceeds as follows: In Step 2 it checks whether $g_{w}^{k} \leqslant \mu$. If so, then $\nexists j \in\left\{j(k)+1, \ldots, o_{w}\right\}$ such that $\sum_{j^{\prime}=j(k)}^{j-1} \alpha_{w}\left(\theta_{j^{\prime}}\right) \geqslant$ $\mu$, hence $U_{k}$ is empty. Otherwise, in Step 3 it searches for the first index $j+1>j(k)$ such that $\tau_{w}\left(\theta_{j}\right)<\tau_{w}\left(\theta_{j+1}\right)$, and in Step 4 it checks whether such an index exists or not. If this index does not exist, then $U_{k}$ is empty. Otherwise, in Step 5 it checks whether $\sum_{j^{\prime}=j(k)}^{j} \alpha_{w}\left(\theta_{j^{\prime}}\right) \geqslant \mu$. If so, then $U_{k}$ is not empty, since the index $j+1$ belongs to $U_{k}$; if not, in Step 3 it searches for the next in$\operatorname{dex} j+1>j(k)$ such that $\tau_{w}\left(\theta_{j}\right)<\tau_{w}\left(\theta_{j+1}\right)$, and so forth. (Notice that, proceeding in this way, it will be $q(w) \leqslant \bar{q}$, since $\bar{q}=\frac{\max \left\{g_{w^{\prime}}^{1} \mid w^{\prime} \in W^{\prime}\right\}}{\mu} \geqslant \frac{g_{w}^{1}}{\mu}=$ $\frac{\sum_{k=1}^{q(w)-1}\left(g_{w}^{k}-g_{w}^{k+1}\right)+g_{w}^{q(w)}}{\mu} \geqslant \frac{(q(w)-1) \mu+g_{w}^{q(w)}}{\mu}=q(w)-1+$ $\frac{g_{w}^{q(w)}}{\mu}>q(w)-1$. $)$

\section{Algorithm 1}

Step 1. Set $g_{w}^{1}=\sum_{\theta \in \Theta_{w}} \alpha_{w}(\theta), i=1$ and $k=1$.

Step 2. If $g_{w}^{k} \leqslant \mu$, set $q(w)=k$ and STOP; otherwise, set $j=i$.

Step 3. Set $j=\max \left\{j^{\prime} \in\left\{j, \ldots, o_{w}\right\} \mid \tau_{w}\left(\theta_{j^{\prime}}\right)=\right.$ $\left.\tau_{w}\left(\theta_{j}\right)\right\}$

Step 4. If $j=o_{w}$, set $q(w)=k$ and STOP. Step 5. If $\sum_{j^{\prime}=i}^{j} \alpha_{w}\left(\theta_{j^{\prime}}\right) \geqslant \mu$, set $k=k+1, u_{w}^{k}=$
$\tau_{w}\left(\theta_{j+1}\right), g_{w}^{k}=g_{w}^{k-1}-\sum_{j^{\prime}=i}^{j} \alpha_{w}\left(\theta_{j^{\prime}}\right), i=j+1$
and go to Step 2; otherwise, set $j=j+1$ and go to Step 3.

In the computational results reported below, each example case has been solved considering several values for $\bar{q}$. For each one of them, the values of $\{q(w)\}_{w \in W^{\prime}}, \quad\left\{u_{w}^{k}\right\}_{w \in W^{\prime}, k \in\{2, \ldots, q(w)\}}$ and $\left\{g_{w}^{k}\right\}_{w \in W^{\prime}, k \in\{1, \ldots, q(w)\}}$ have been computed by applying Algorithm 1. We have taken $M_{w}^{k}=\bar{t}^{\prime}(w)+\bar{t}_{n-1}^{\prime}$ 
$u_{w}^{k} \quad \forall w \in W^{\prime}, \forall k \in\{1, \ldots, q(w)\}$ (see Section 3); we have considered some other settings for the values of $\left\{M_{w}^{k}\right\}_{w \in W^{\prime}, k \in\{1, \ldots, q(w)\}}$, but the best general computational results have been obtained with these ones.

The implementation platform has been Microsoft Visual C++ 2008, CPLEX v12.3, and Intel Core i5$2300 \mathrm{CPU}, 2.80 \mathrm{GHz}, 8.00 \mathrm{~Gb}$ RAM.

In order to solve the model stated in Section 3, we have run the CPLEX mixed integer optimizer by using the default rules except that the relative and absolute optimality tolerances have been set to zero, a time limit of two hours has been imposed, and, in the branching process, the priorities for the variables $\left\{x_{i j}\right\}_{\{i, j\} \in E}$ and $\left\{p_{w}^{k}\right\}_{w \in W^{\prime}, k \in\{1, \ldots, q(w)\}}$ have been set to 1 and 2, respectively (we have considered many other settings for the priority values, but the best general computational results have been obtained with these ones).

Tables 4-8 show, respectively, the computational results obtained for $C 1, \ldots, C 5$ by considering several values for $\bar{q}$. They illustrate how the computational effort required for solving the model stated in Section 3 increases as the values of $n$ and $m$ increase.

The columns headed " $z^{*}$, "Nodes" and " $M$. time" give, respectively, the value of the objective function at the optimal or incumbent solution to the model stated in Section 3, the number of branchand-cut nodes evaluated for solving that model and the related CPU time expressed in seconds. For the optimal or incumbent solution obtained, the columns headed " $\bar{s}$ " and " $C C$ " give, respectively, the number of non-key stations to be constructed, and the construction cost of the related network.
Table 4. Computational results for $C 1$

\begin{tabular}{rrrrrc}
\hline $\bar{q}$ & \multicolumn{1}{c}{$z^{*}$} & Nodes & M. time & $\bar{s}$ & $C C$ \\
\hline 1 & 4094 & 19 & 3 & 42 & 5856.55 \\
2 & 6810 & 107 & 3 & 42 & 5991.82 \\
3 & 10047 & 379 & 3 & 42 & 5965.13 \\
4 & 11947 & 186 & 2 & 42 & 5979.18 \\
5 & 12891 & 520 & 4 & 40 & 5783.16 \\
6 & 13604 & 6 & 2 & 41 & 5884.32 \\
7 & 14106 & 255 & 2 & 42 & 5936.75 \\
8 & 14456 & 325 & 2 & 42 & 5937.76 \\
9 & 14556 & 501 & 3 & 42 & 5926.11 \\
10 & 14725 & 1261 & 12 & 41 & 5864.54 \\
11 & 14927 & 1415 & 13 & 40 & 5802.23 \\
12 & 15071 & 873 & 10 & 41 & 5886.63 \\
13 & 15123 & 945 & 11 & 41 & 5886.63 \\
14 & 15183 & 602 & 8 & 42 & 5930.35 \\
15 & 15253 & 3473 & 20 & 42 & 5930.35 \\
16 & 15407 & 555 & 9 & 41 & 5886.63 \\
17 & 15418 & 5223 & 28 & 41 & 5886.63 \\
18 & 15418 & 5223 & 29 & 41 & 5886.63 \\
19 & 15555 & 1555 & 14 & 42 & 5994.53 \\
20 & 15603 & 4302 & 25 & 42 & 5994.53 \\
\hline
\end{tabular}

Table 5. Computational results for $C 2$

\begin{tabular}{rrrrcc}
\hline $\bar{q}$ & \multicolumn{1}{c}{$z^{*}$} & Nodes & M. time & $\bar{s}$ & $C C$ \\
\hline 1 & 4283 & 253 & 28 & 54 & 7768.57 \\
2 & 6929 & 500 & 38 & 55 & 7985.83 \\
3 & 11294 & 6042 & 46 & 56 & 7911.91 \\
4 & 13846 & 1833 & 41 & 55 & 7924.79 \\
5 & 15581 & 2982 & 37 & 55 & 7931.32 \\
6 & 16713 & 525 & 28 & 55 & 7915.38 \\
7 & 17461 & 3384 & 45 & 55 & 7874.69 \\
8 & 18009 & 1568 & 30 & 55 & 7874.69 \\
9 & 18326 & 2701 & 47 & 55 & 7997.01 \\
10 & 18530 & 1447 & 32 & 55 & 7997.01 \\
11 & 18938 & 516 & 26 & 55 & 7997.01 \\
12 & 19178 & 546 & 24 & 55 & 7997.01 \\
13 & 19362 & 586 & 15 & 55 & 7997.01 \\
14 & 19447 & 2298 & 33 & 55 & 7987.60 \\
15 & 19565 & 3161 & 25 & 55 & 7966.96 \\
16 & 19681 & 6950 & 43 & 55 & 7997.01 \\
17 & 19734 & 3532 & 85 & 55 & 7966.96 \\
18 & 19734 & 3532 & 85 & 55 & 7966.96 \\
19 & 19910 & 2465 & 50 & 55 & 7966.96 \\
20 & 19910 & 2465 & 51 & 55 & 7966.96 \\
\hline
\end{tabular}


Table 6. Computational results for $C 3$

\begin{tabular}{rrrrcc}
\hline $\bar{q}$ & \multicolumn{1}{c}{$z^{*}$} & Nodes & M. time & $\bar{s}$ & $C C$ \\
\hline 1 & 5370 & 534 & 122 & 96 & 12881.4 \\
2 & 9108 & 519 & 113 & 97 & 12995.7 \\
3 & 15669 & 548 & 123 & 97 & 12969.0 \\
4 & 19872 & 4821 & 404 & 87 & 11912.1 \\
5 & 22507 & 8403 & 140 & 95 & 12783.8 \\
6 & 24399 & 542 & 86 & 95 & 12725.8 \\
7 & 25464 & 8288 & 132 & 96 & 12837.5 \\
8 & 26260 & 1336 & 121 & 96 & 12838.8 \\
9 & 26790 & 566 & 105 & 97 & 12949.5 \\
10 & 27332 & 1344 & 113 & 97 & 12973.6 \\
11 & 27618 & 1239 & 85 & 96 & 12793.1 \\
12 & 28172 & 2254 & 93 & 95 & 12725.8 \\
13 & 28575 & 2700 & 126 & 95 & 12747.6 \\
14 & 28692 & 501 & 77 & 96 & 12858.2 \\
15 & 28919 & 1281 & 98 & 95 & 12747.6 \\
16 & 29106 & 1045 & 92 & 96 & 12845.5 \\
17 & 29308 & 977 & 84 & 96 & 12858.2 \\
18 & 29308 & 977 & 84 & 96 & 12858.2 \\
19 & 29458 & 1960 & 104 & 95 & 12773.3 \\
20 & 29458 & 1960 & 105 & 95 & 12773.3 \\
\hline
\end{tabular}

Table 7. Computational results for $C 4$

\begin{tabular}{rrrrcc}
\hline $\bar{q}$ & \multicolumn{1}{c}{$z^{*}$} & Nodes & M. time & $\bar{s}$ & $C C$ \\
\hline 1 & 8154 & 16348 & 542 & 120 & 15864.3 \\
2 & 11943 & 2160 & 429 & 121 & 15994.9 \\
3 & 19097 & 4487 & 291 & 121 & 15977.6 \\
4 & 24083 & 6126 & 749 & 117 & 15434.6 \\
5 & 26849 & 1430 & 292 & 120 & 15977.1 \\
6 & 28810 & 8541 & 364 & 121 & 15987.3 \\
7 & 30068 & 1218 & 336 & 118 & 15741.4 \\
8 & 30987 & 31696 & 1135 & 121 & 15994.9 \\
9 & 31933 & 6919 & 375 & 118 & 15723.2 \\
10 & 32427 & 3878 & 349 & 120 & 15859.4 \\
11 & 32682 & 3006 & 266 & 119 & 15837.3 \\
12 & 33204 & 10457 & 409 & 120 & 15939.7 \\
13 & 33528 & 3675 & 358 & 120 & 15994.8 \\
14 & 33862 & 10069 & 366 & 120 & 15962.8 \\
15 & 34149 & 8959 & 515 & 120 & 15971.1 \\
16 & 34149 & 8959 & 515 & 120 & 15971.1 \\
17 & 34389 & 10512 & 436 & 121 & 15975.7 \\
18 & 34529 & 11213 & 479 & 121 & 15977.5 \\
19 & 34529 & 11213 & 479 & 121 & 15977.5 \\
20 & 34808 & 6971 & 359 & 118 & 15690.7 \\
\hline
\end{tabular}

Table 8. Computational results for $C 5$

\begin{tabular}{rcrrcc}
\hline $\bar{q}$ & $z^{*}$ & Nodes & M. time & $\bar{s}$ & $C C$ \\
\hline 1 & 13862 & 1139 & 781 & 124 & 17756.5 \\
2 & 15633 & 32338 & 1100 & 126 & 17917.9 \\
3 & 21289 & 299276 & 5129 & 126 & 17986.2 \\
4 & 26059 & 83434 & 3664 & 124 & 17759.2 \\
5 & 29413 & 2877 & 886 & 124 & 17662.9 \\
6 & 32120 & 17613 & 1221 & 126 & 17951.8 \\
7 & 33641 & 2275 & 654 & 126 & 17955.4 \\
8 & 34754 & 32684 & 1137 & 127 & 17987.4 \\
9 & 35906 & 5747 & 929 & 126 & 17956.5 \\
10 & 36488 & 11022 & 1166 & 126 & 17997.2 \\
11 & 37172 & 15633 & 878 & 126 & 17944.2 \\
12 & 37537 & 2585 & 491 & 126 & 17960.8 \\
13 & 37867 & 7182 & 605 & 126 & 17865.6 \\
14 & 38254 & 18250 & 934 & 126 & 17919.9 \\
15 & 38516 & 6924 & 630 & 126 & 17960.3 \\
16 & 38882 & 59100 & 5732 & 126 & 17849.4 \\
17 & 39141 & 113270 & 7207 & 126 & 17960.3 \\
18 & 39141 & 112074 & 7205 & 126 & 17960.3 \\
19 & 39413 & 44511 & 5814 & 126 & 17971.1 \\
20 & 39413 & 44511 & 5818 & 126 & 17971.1 \\
\hline
\end{tabular}

In spite of the fact that the greater the value of $\bar{q}$, the larger the dimension of the model stated in Section 3, it can be observed from Tables 4-8 that the values of " $M$. time" do not follow an increasing trend as the value of $\bar{q}$ increases. In any case, bearing in mind the size of the instances we are dealing with, the computational effort required for solving the model stated in Section 3 can be considered quite small (notice that $C 5$ is the unique example case where the imposed time limit has been reached, and this has occurred only for $\bar{q} \in\{17,18\}$ ).

If we had considered the all-or-nothing model for the potential users' behavior from Marín ${ }^{8}$, which was also considered in Escudero and Muñoz ${ }^{12,13}$, then we would have obtained the value of $z^{*}$ for $\bar{q}=1$ (see Section 3), which is less than the values of $z^{*}$ for the rest of the values considered for $\bar{q}$.

\section{Conclusions and Future Research}

We have presented the first stage of a two-stage approach for designing rapid transit networks, which is based on the first stage of another approach that we described elsewhere. 
Whereas most of the procedures that can be found in the literature compute their objective function values by means of a given static O-D matrix, we have proposed to perform a survey amongst the potential users of the rapid transit network. The survey results make it possible to consider each potential user's behavior individually, which allows to compute our objective function value (i.e., the expected number of trips through the rapid transit network) in a more accurate way.

The model stated in Section 3 for selecting the stations and links to be constructed without exceeding the available budget does not take into account the transfer times for the users, since no line design is available at this stage. These transfer times will be considered in a subsequent phase.

The computational experience has shown that, in the first stage of our approach, we can handle medium and medium-large size instances within a relatively small time.

In order to deal with large size instances, we are working on preprocessing techniques for solving the model stated in Section 3. In case that this model could not be solved to optimality, the stations and links to be constructed would be selected according to the incumbent solution obtained. We are also working on the problem of determining the headways for the lines.

\section{Acknowledgments}

This research was supported in part by the grants MTM2009-14087-C04-01 and TIN200907901 from Ministerio de Ciencia e Innovación, Spain.

The authors are grateful to two anonymous reviewers for their helpful comments on the first version of this paper.

\section{Appendix A Notation in Section 2}

Below we provide a summarized list containing the notation considered in Section 2, in order of appearance.
$V \quad$ set of key station locations $(V=\{1, \ldots, n\})$

$E \quad$ set of pairs of key station locations that can potentially be linked $(E=\{\{i, j\} \in V \times V \mid$ $i \neq j$ and it is possible to link $i$ and $j\}$ )

$m=|E|$

$G=(V, E)$

$a_{i} \quad$ cost of constructing a key station at location $i$

$\Gamma(i)$ set of key station locations that can be linked to location $i$

$d_{i j} \quad$ length (in kms.) of link $\{i, j\}$

$s_{i j} \quad$ number of non-key station locations on link $\{i, j\}$

$c_{i j} \quad$ cost of linking $i$ and $j$

$b \quad$ available budget for constructing the rapid transit network

$\bar{v} \quad$ average velocity (in $\mathrm{kms} / \mathrm{h}$ ) of the network's vehicles

$\bar{t}(i)$ average time (in min.) required for going between the entrance of the key station located at $i$ and its platform

$\bar{t}_{a}$ average interarrival time (in min.) of the vehicles at each station

$\bar{t}_{s} \quad$ average dwell time (in min.) of the vehicles at each station

$\bar{t}_{r} \quad$ average time (in min.) for making a transfer

$W=\{(i, j) \in V \times V \mid i<j\}$

$w=\left(e_{w}, e_{w}^{\prime}\right) \quad \forall w \in W$

$\Theta \quad$ set of surveyed people

$\alpha_{w}(\theta)$ number of trips in a working week that the surveyee $\theta$ plans to take between $e_{w}$ and $e_{w}^{\prime}$ during the hours of operation of the rapid transit network

$W_{\theta}=\left\{w \in W \mid \alpha_{w}(\theta)>0\right\} \quad \forall \theta \in \Theta$

$d_{e_{w} e_{w}^{\prime}}$ Euclidean distance (in kms.) between $e_{w}$ and $e_{w}^{\prime}$ if $\left\{e_{w}, e_{w}^{\prime}\right\} \notin E$

$\widehat{t}_{w}=\bar{t}\left(e_{w}\right)+\frac{\bar{t}_{a}}{2}+\frac{60}{\bar{v}} d_{e_{w} e_{w}^{\prime}}+\bar{t}\left(e_{w}^{\prime}\right) \quad \forall w \in W$

$\tau_{w}(\theta)$ maximum amount of time (in min.) that the surveyee $\theta$ is willing to spend for traveling between $e_{w}$ and $e_{w}^{\prime}$ 


$$
\begin{aligned}
& W^{\prime}=\bigcup_{\theta \in \Theta} W_{\theta} \\
& \Theta_{w}=\left\{\theta \in \Theta \mid \alpha_{w}(\theta)>0\right\} \quad \forall w \in W^{\prime} \\
& q(w) \text { number of time intervals utilized to estimate } \\
& \Theta_{w}^{k}=\left\{\theta \in \Theta_{w} \mid \tau_{w}(\theta) \geqslant u_{w}^{k}\right\} \quad \forall w \in W^{\prime}, \\
& \forall k \in\{1, \ldots, q(w)\} \\
& g_{w}^{k}=\sum_{\theta \in \Theta_{w}^{k}} \alpha_{w}(\theta) \quad \forall w \in W^{\prime}, \forall k \in\{1, \ldots, q(w)\}
\end{aligned}
$$

\section{Appendix B Notation in Section 3}

Below we provide a summarized list containing the definition of the variables and the rest of notation considered in Section 3, in order of appearance.

\section{Variables:}

$$
\begin{aligned}
& x_{i j}=\left\{\begin{array}{ll}
1 & \text { if } i \text { and } j \text { are linked } \\
0 & \text { otherwise }
\end{array} \forall\{i, j\} \in E\right. \\
& \gamma_{i}= \begin{cases}1 & \text { if } \sum_{j \in \Gamma(i), j>i} x_{i j}+\sum_{j \in \Gamma(i), j<i} x_{j i} \text { is odd } \\
0 & \text { otherwise }\end{cases}
\end{aligned}
$$

$$
\forall i \in V
$$

$$
\Delta_{i} \in\{0, \ldots, r(i)\} \quad \forall i \in V
$$$$
f_{i j}^{w}= \begin{cases}1 & \begin{array}{l}
\text { if the preliminary route for traveling } \\
\text { between } e_{w} \text { and } e_{w}^{\prime} \\
\text { otherwise }
\end{array}\end{cases}
$$

$$
\forall w \in W, \forall\{i, j\} \in E
$$

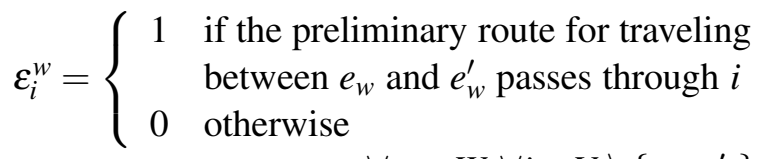

$$
\begin{aligned}
& \forall w \in W, \forall i \in V \backslash\left\{e_{w}, e_{w}^{\prime}\right\} \\
& p_{w}^{k} \in\{0,1\} \quad \forall w \in W^{\prime}, \forall k \in\{1, \ldots, q(w)\}
\end{aligned}
$$

\section{Rest of notation:}

$r(i)=\left\{\begin{array}{ll}\frac{|\Gamma(i)|}{2} & \text { if }|\Gamma(i)| \text { is even } \\ \frac{|\Gamma(i)|-1}{2} & \text { if }|\Gamma(i)| \text { is odd }\end{array} \quad \forall i \in V\right.$

$\bar{t}^{\prime}(w)=\bar{t}\left(e_{w}\right)+\frac{\bar{t}_{a}}{2}-\bar{t}_{s}+\bar{t}\left(e_{w}^{\prime}\right) \quad \forall w \in W^{\prime}$

$\bar{t}_{i j}^{\prime}=\frac{60}{\bar{v}} d_{i j}+\bar{t}_{s}\left(s_{i j}+1\right) \quad \forall\{i, j\} \in E$

$M_{w}^{k} \quad$ upper bound on the value of $\bar{t}^{\prime}(w)+$ $\sum_{\{i, j\} \in E} \bar{t}_{i j}^{\prime} f_{i j}^{w}-u_{w}^{k}$

$\bar{t}_{n-1}^{\prime}$ sum of the $n-1$ greatest values in $\left\{\bar{t}_{i j}^{\prime}\right\}_{\{i, j\} \in E}$

\section{References}

1. M. T. Claessens, N. M. van Dijk and P. J. Zwaneveld, "Cost optimal allocation of rail passenger lines," Eur. J. Oper. Res., 110, 474-489 (1998).

2. M. R. Bussieck, T. Lindner and M. E. Lübbecke, "A fast algorithm for near cost optimal line plans," Math. Methods Oper. Res., 59, 205-220 (2004).

3. J. W. Goossens, S. van Hoesel and L. Kroon, "A branch-and-cut approach for solving railway lineplanning problems," Transp. Sci., 38, 379-393 (2004).

4. C. E. Mandl, "Evaluation and optimization of urban public transportation networks," Eur. J. Oper. Res., 5, 396-404 (1980).

5. M. H. Baaj and H. S. Mahmassani, "An AI-based approach for transit route system planning and design," J. Adv. Transp., 25, 187-209 (1991).

6. M. R. Bussieck, P. Kreuzer and U. T. Zimmermann, "Optimal lines for railway systems," Eur. J. Oper. Res., 96, 54-63 (1997).

7. J. F. Guan, H. Yang and S. C. Wirasinghe, "Simultaneous optimization of transit line configuration and passenger line assignment," Transp. Res. Part B, 40, 885-902 (2006).

8. Á. Marín, "An extension to rapid transit network design problem," Top, 15, 231-241 (2007).

9. G. Laporte, J. A. Mesa and F. Perea, "A game theoretic framework for the robust railway transit network design problem," Transp. Res. Part B, 44, 447-459 (2010).

10. G. Laporte, Á. Marín, J. A. Mesa and F. Perea, "Designing robust rapid transit networks with alternative routes," J. Adv. Transp., 45, 54-65 (2011).

11. Á. Marín and R. García-Ródenas, "Location of infrastructure in urban railway networks," Comput. Oper. Res., 36, 1461-1477 (2009). 
12. L. F. Escudero and S. Muñoz, "An approach for solving a modification of the extended rapid transit network design problem," Top , 17, 320-334 (2009).

13. L. F. Escudero and S. Muñoz, "On designing connected rapid transit networks reducing the number of transfers," RAIRO Oper. Res., 45, 315-338 (2011).

14. J. G. Wardrop, "Some theoretical aspects of road traffic research," ICE Proc.: Eng. Div., 1, 325-362 (1952).

15. L. F. Escudero and S. Muñoz, "Designing rapid transit networks from the results of a survey," Working paper, Universidad Complutense de Madrid, Spain (2010) (available at http://eprints.ucm.es/10412/).

16. Z. Gao, J. Wu and H. Sun, "Solution algorithm for the bi-level discrete network design problem," Transp. Res. Part B, 39, 479-495 (2005).

17. H. Zhang and Z. Gao, "Bilevel programming model and solution method for mixed transportation network design problem," J. Syst. Sci. Complex., 22, 446-459 (2009).

18. G. Laporte, Á. Marín, J. A. Mesa and F. A. Ortega, "An integrated methodology for the rapid transit network design problem," In: F. Geraets, L. Kroon, A. Schoebel, D. Wagner and C. D. Zaroliagis (eds), "Algorithmic methods for railway optimization," Lect. Notes Comput. Sci., vol 4359, Springer, Berlin, 187199 (2007). 